Cahiers $d u$ MONDE RUSSE

\section{Cahiers du monde russe}

Russie - Empire russe - Union soviétique et États indépendants

$51 / 4 \mid 2010$

Sciences humaines et sociales en Russie à l'Âge d'argent

\title{
Le formalisme russe et ses sources
}

Quelques considérations de méthode

The sources of Russian Formalism: Methodological considerations

\section{Catherine Depretto}

\section{(2) OpenEdition}

\section{Journals}

Édition électronique

URL : https://journals.openedition.org/monderusse/9208

DOI : 10.4000/monderusse. 9208

ISSN : $1777-5388$

Éditeur

Éditions de l'EHESS

Édition imprimée

Date de publication : 25 novembre 2010

Pagination : $565-579$

ISBN : 978-2-7132-2316-7

ISSN : $1252-6576$

Référence électronique

Catherine Depretto, «Le formalisme russe et ses sources », Cahiers du monde russe [En ligne], 51/4 I 2010, mis en ligne le 20 décembre 2013, consulté le 03 septembre 2022. URL : http://

journals.openedition.org/monderusse/9208; DOI : https://doi.org/10.4000/monderusse.9208

Ce document a été généré automatiquement le 3 septembre 2022.

Tous droits réservés 


\title{
Le formalisme russe et ses sources
}

\author{
Quelques considérations de méthode \\ The sources of Russian Formalism: Methodological considerations
}

Catherine Depretto

\section{Le contexte européen du formalisme russe}

1 Le formalisme russe a été redécouvert dans un contexte bien spécifique, celui de la poussée structuraliste en sciences humaines, dans les années 1960. Devant le caractère exceptionnel de cet ensemble de textes qui tranchait par rapport à la grisaille de la critique marxiste-léniniste soviétique, on s'est d'abord peu intéressé aux sources intellectuelles du mouvement, sauf de façon très générale, en évoquant ses liens avec la Poétique d'Aristote ou l'idéalisme kantien; on a aussi parlé de ses prédécesseurs slaves, Aleksandr Potebnja et Aleksandr Veselovskij ${ }^{1}$. La principale grille de lecture, adoptée à l'époque en Occident, consistait à montrer en quoi le structuralisme était un dépassement du formalisme ${ }^{2}$. De fait, comme entre la date de conception des principaux textes formalistes et la date de leur redécouverte il s'était écoulé quasiment cinquante ans, les sources immédiates du mouvement étaient oubliées; le contexte scientifique initial de leur rédaction appartenait à l'histoire et avait besoin d'être reconstitué. La même chose s'est produite avec le Rabelais de Bahtin, conçu dans les années 1930, mais lu dans les années 1970. L'importance de la querelle franco-allemande Lefranc-Spitzer pour comprendre le sens de cet ouvrage n'a été mise en évidence que très récemment par Irina Popova ${ }^{3}$.

2 Aussi, après une première phase de reconstitution de l'histoire russe du formalisme, s'est développée récemment une perspective «inclusive», qui cherche à insérer le mouvement dans un contexte européen plus large ; on doit les principales avancées en ce domaine à Aleksandr Dmitriev, Patrick Sériot, Ilona Svetlikova, Serguei Tchougounnikov, Céline Trautmann-Waller, Sergej Zenkin ${ }^{4}$. On rappelle néanmoins que dans La Méthode formelle en littérature : introduction à une poétique sociologique (1928), Pavel Medvedev posait déjà le problème en ces termes : 
Si l'on adopte une perspective historique plus large, la méthode formelle russe n'est que l'une des ramifications d'un mouvement formel qui est commun à toute l'Europe dans le domaine des sciences de l'art. ${ }^{5}$

\section{De l'importance des sources germaniques}

3 À de rares exceptions près, le contexte scientifique considéré comme déterminant pour comprendre le formalisme russe est la tradition germanique ; ce qui n'a rien d'étonnant, compte tenu de la position dominante de la philologie allemande dans le champ et du fait que Potebnja, Veselovskij étaient déjà eux-mêmes marqués par la recherche germanique.

Dans cet ensemble, il est possible de distinguer plusieurs composantes :

- des philosophes comme Wilhelm Dilthey (1833-1911), des plasticiens, théoriciens de l'art, comme Heinrich Wölfflin (1864-1945') ou Wilhelm Wörringer (1881-1965) ;

- des théoriciens de la littérature comme Wilhelm Dibelius (1876-1931), Oskar Walzel (1864-1944);

- des linguistes, représentants de la philologie auditive (phonétique acoustique), Eduard Sievers (1850-1932) et Franz Saran (1866-1931), membres d'honneur, comme Walzel, de l'Institut d'État d'histoire de l'art (Gosudarstvennyj Institut istorii iskusstv, GIII), le bastion du formalisme à Leningrad dans les années 1920 ;

- enfin des psychologues comme Johann Friedrich Herbart (1776-1841) ou Wilhelm Wundt (1832-1920).

Effectivement, l'influence de l'Ohrenphilologie (Sievers et Saran) est incontestable lorsqu'on aborde les travaux formalistes sur la composante sonore du vers, ceux de Boris Ejhenbaum (Melodika russkogo liričeskogo stixa [Mélodie du vers lyrique russe], 1922 $)$, de Sergej Bernštejn (1892-1970) qui enregistrait systématiquement les voix des poètes et des écrivains), de Jurij Tynjanov (Problema stihotvornogo jazyka [Problème de la langue du vers], 1924), même si, dans ce dernier ouvrage en particulier, est également engagée une remise en cause de certaines conclusions de la philologie auditive. L'Institut du mot vivant (Institut živogo slova9) qui se met en place à Petrograd en 1919 et auquel collabore Bernštejn n'est pas sans subir l'influence de l'Ohrenphilologie. Une figure emblématique de l'influence allemande en terrain russe est sans doute Viktor Žirmunskij (1891-1971). Germaniste, il a séjourné en Allemagne pendant ses études, est ami de Walzel, qu'il fait traduire en russe ${ }^{10}$, comme d'autres auteurs germaniques (cf. Problemy literaturnoj formy [Problèmes de la forme littéraire], 1928 ${ }^{11}$ ); il cherche aussi à populariser le formalisme à l'étranger, en Allemagne, grâce à un article de synthèse sur le mouvement qui paraît en 1925 dans la revue d'un autre de ses amis, Max Vasmer ${ }^{12}$. Quant à son premier ouvrage, Nemeckij romantizm $i$ sovremennaja mistika ([Le Romantisme allemand et la mystique contemporaine], 1914), il est écrit sous l'influence directe de Wölfflin et de Walzel, et reprend la thèse concernant les deux formes qui structurent la littérature, la métaphore caractéristique du romantisme et du symbolisme, la métonymie, caractéristique du classicisme, du réalisme et de l'acméisme. À ces sources, on peut encore ajouter Broder Christiansen, auquel les formalistes sont redevables de la notion de « dominante » et dont la lecture fait grande impression sur Boris Ejhenbaum ${ }^{13}$. 


\section{Sources françaises}

6 Si les sources allemandes sont incontestables, les sources françaises le sont tout autant ${ }^{14}$, à commencer par l'explication de texte à la Lanson, les travaux de Brunetière, ceux de Maurice Grammont sur le vers, tout comme sont décisives la phonétique expérimentale de l'Abbé Rousselot (1846-1924), la stylistique de Bally et Sechehaye, la sémantique de Bréal, et surtout la linguistique de Saussure : le point 6 du manifeste commun JakobsonTynjanov, "Problèmes des études littéraires et linguistiques» (1928) mentionne explicitement l'opposition "parole et langue » en renvoyant à «l'école de Genève ». Cette même opposition nourrit très certainement la façon dont Tynjanov envisage l'évolution littéraire. Néanmoins la question du saussurisme en Russie et de son influence sur le formalisme reste complexe. Même si la pensée de Saussure occupe une place importante dans les débats de l'époque et en particulier dans les milieux formalistes (Sergej Bernštejn à Petrograd, Aleksandr Romm, Grigorij Vinokur à Moscou), on sait qu'il n'y avait pratiquement pas d'exemplaires du Cours de linguistique générale en Russie et que la traduction du livre, entreprise par A. Romm, fut interrompue en raison de la froideur manifestée par Bally et Sechehaye à l'égard de cette entreprise ${ }^{15}$. On ne doit pas oublier non plus Bergson et sa théorie du rire ou sa conception du temps, pour comprendre "l'évolution littéraire " selon Tynjanov ${ }^{16}$. Récemment à cet ensemble déjà impressionnant est venue s'ajouter, en ce qui concerne la sémantique poétique de Tynjanov, la psychologie de Théodule Ribot. C'est ce qu'établit de manière convaincante Ilona Svetlikova, montrant en particulier que la langue de Tynjanov, si difficile à comprendre dans Problème de la langue du vers, s'éclaire si l'on comprend qu'elle s'inspire d'une terminologie psychologique. Par rapport aux sources allemandes, les sources françaises sont un peu d'une autre nature; elles sont plus morphologiques que philosophiques, plus linguistiques qu'esthétiques, mais ne sont pas moins importantes.

\section{Un formalisme ou des formalismes?}

7 Si l'on cherche à faire la synthèse de ce qui a été fait jusqu'à présent dans cette question des sources, on se trouve face à une suite de noms qui montrent que certains des formalistes étaient des gens très cultivés, bien au fait de ce qui paraissait à l'époque. Il est difficile de trouver dans ces sources une clé qui rendrait plus compréhensible le sens de leur travail. Ce qui est vrai pour certains formalistes ne l'est pas pour d'autres et la plupart des travaux sur le formalisme et ses sources traitent, en réalité, des sources de l'un de ses représentants: Sergueï Tchougounnikov s'intéresse surtout à Polivanov et Jakobson, Ilona Svetlikova à Brik, Tynjanov. On se heurte ici à un élément fondamental et bien connu de l'histoire du formalisme russe. Le mouvement renvoie à un ensemble de chercheurs, unis par des gestes fondamentaux, des principes, mais gardant chacun leur individualité et ayant chacun apporté quelque chose de spécifique. Il n'y a jamais eu un corps de doctrine constitué, de thèses à suivre à la lettre, en un mot de "doctrine formaliste", si bien qu'il est sans doute impossible de vouloir apporter une réponse globale à la question des sources. Du vivant même des formalistes, cette question était le plus souvent objet de polémique, ce qui a largement contribué à obscurcir le débat et les a peu encouragés à expliciter les influences subies, les emprunts réalisés. On leur a beaucoup reproché, par exemple, de rompre avec la tradition russe de la critique pour laquelle la littérature «était avant tout la tribune d'où s'élevaient des propos 
didactiques» et d'introduire, en privilégiant les questions formelles, une approche étrangère ${ }^{17}$. D'un autre côté, on ne s'est pas privé de dire qu'ils n'avaient rien inventé et répétaient des vérités établies depuis longtemps en Europe occidentale.

Les études récentes n'ont fait qu'élargir et diversifier le cercle de ceux qui sont concernés par le formalisme en Russie, ce qui tend encore à complexifier la question des sources. On connaît mieux, par exemple, le formalisme de Moscou (Grigorij Vinokur, Boris Jarho) ${ }^{18}$, ainsi que le formalisme de la seconde génération, celle des élèves (Boris Buhštab, Lidija Ginzburg) ${ }^{19}$. Le cercle des " précurseurs » russes immédiats s'est aussi considérablement élargi, il n'inclut pas seulement Andrej Belyj et ses travaux sur le tétramètre iambique et le rythme, mais aussi Sergej Bobrov (1889-1971), Valerian Čudovskij (1891-1938?), Nikolaj Nedobrovo (1882-1919), Georgij Šengeli (1894-1956) ${ }^{20}$ ou Mihail Lopatto (1892-1981), Georgij Maslov (1895-1920). Cette diversité du formalisme en Russie ne signifie pas que la question de ses sources étrangères ne doive pas être posée, mais elle doit l'être en tenant compte de cette diversité comme de l'évolution de ses principaux représentants. D'autre part, une influence étrangère ne s'exprime pas forcément de manière directe, par la lecture d'ouvrages originaux, mais peut refléter, de manière plus diffuse, un climat intellectuel, une atmosphère, certes nourris de sources étrangères, mais s'exerçant dans un cadre national. Et de ce point de vue, il semble essentiel d'insister sur l'existence d'un terreau national, propice au développement d'une approche formaliste de la littérature, le département d'études romanes et germaniques de l'université de Saint-Pétersbourg.

\section{Un cadre national pour des sources étrangères : le département d'études romanes et germaniques de l'université de Saint-Pétersbourg}

9 Fondé en 1884 par le comparatiste Aleksandr Veselovskij (1838-1906), ce département représentait avant la révolution quelque chose d'original en Russie ${ }^{21}$; il se caractérisait par une ouverture affirmée en direction de la culture européenne, et en particulier de la culture romane. Cette ouverture était relayée par la Société néo-philologique (Neofilologičeskoe ob̌̌čestvo), organisée par Aleksandr Veselovskij en 1885 qui comptait parmi ses membres d'honneur Frédéric Mistral, mais aussi l'abbé Rousselot, Louis Leger, Antoine Thomas, Sievers, les Italiens Alessandro D'Ancona et Pio Rajna; pendant les trente années de son existence, elle constitua un foyer intellectuel important ${ }^{22}$. Parmi les formalistes, on trouve plusieurs anciens élèves de ce département, Viktor Žirmunskij et Boris Ejhenbaum au premier chef, auxquels on peut ajouter les romanistes Vladimir Šklovskij et Konstantin Močul'skij ${ }^{23}$, jamais présents dans les histoires du mouvement et qui pourtant y ont été associés.

\section{Deux formalistes oubliés : Vladimir Šklovskij et Konstantin Močul'skij}

Frère aîné de Viktor Šklovskij (1893-1984), Vladimir Šklovskij (1889-1937) a terminé ses études de philologie romane en 1910, avec une spécialité assez exotique pour la Russie de l'époque, l'espagnol. Il commence, ensuite, à enseigner le français à l'Académie ecclésiastique jusqu'à sa fermeture en 1919, puis à l'Institut de théologie ; il avait fait des 
séjours à l'étranger, comme étudiant en 1908, 1911, 1913 (Grenoble, Besançon, Paris) et en 1914, comme correspondant du journal L'École et la vie. Son premier ouvrage Neofilologičeskie opyty 1915 porte sur un poète religieux italien du XIII e siècle, Fra Jacopone da Todi. Il était certainement destiné à une brillante carrière ; sa famille fondait sur lui de grands espoirs; homme de cabinet, grand érudit, remarquable polyglotte, il connaissait une quinzaine de langues vivantes et anciennes et travaillait tout le temps (ses trajets en tramway lui servaient à apprendre le portugais). Il était aussi très pieux (orthodoxe) et c'est la raison principale des arrestations régulières ${ }^{24}$ et des peines de camp (Solovki, Belomor ${ }^{25}$ ) dont est ponctuée son existence à partir de 1918 jusqu'à son exécution en $1937^{26}$. Bien que réhabilité en 1963 , il n'a été possible de travailler sur lui qu'à partir de la perestroïka, si bien que sa vie et ses travaux commencent à peine à sortir de l'ombre, grâce aux recherches de Larisa Stepanova en particulier. Aussi ne connaîtrat-on sans doute qu'une infime partie de son œuvre; ses archives sont dispersées dans différents fonds et ce qui reste est le plus souvent difficilement exploitable. De son vivant, après quelques publications, il n'a pu faire paraître, pour l'essentiel, que des articles.

11 Vladimir Šklovskij a laissé un nom dans les études romanes pour avoir publié en 1922 la première traduction russe de De vulgari eloquio ${ }^{27}$ de Dante, texte capital posant en quelque sorte les fondements de la philologie italienne (quelle langue?, quelle métrique ?); il avait plusieurs études sur la littérature italienne en préparation dont un «Dante philologue » et, dans ses archives, il reste un manuscrit difficilement exploitable d'un grand travail sur la «Romania» (origine et signification du terme), entrepris dans les années $1930^{28}$. Il était très intéressé par les problèmes de traduction et la constitution des dictionnaires: dans ses archives, il reste également un manuscrit sur ces questions qui montre une bonne familiarité avec la culture française classique. Parmi ses autres travaux, non retrouvés, on note un "A.N. Veselovskij et sa poétique historique », des études sur la langue française contemporaine, en particulier sur l'argot des tranchées, sur la littérature espagnole (il se considérait comme l'élève de l'hispaniste D.K. Petrov ${ }^{29}$ ).

12 Vladimir Šklovskij peut aussi être considéré comme un des premiers formalistes russes; il a participé aux Recueils de la société d'étude du langage poétique (Opojaz), avec des traductions du Danois Kristoffer Nyrop (1858-1931), « Le son et sa signification », extrait de sa Grammaire historique de la langue française, 1913, de Maurice Grammont (1866-1946), le Vers français, 1913, et une analyse des travaux de Sievers ${ }^{30}$. Ces traductions montrent son intérêt pour l'aspect sonore de la création verbale, un des thèmes du premier formalisme; c'est tout naturellement qu'il suit les travaux de Sergej Bernštejn en ce domaine. Ses genres favoris sont les genres lus à haute voix, théâtre et poésie; il est l'un des premiers à aborder le thème de l'art de la conversation dans les salons français. C'est lui qui connaissait le mieux les publications occidentales (cf. son « Panorama des travaux de philologie romane ») et, pour cette raison, les membres de l'Opojaz tenaient à sa collaboration. Viktor Šklovskij aurait déclaré à son secrétaire Aleksandr Galuškin qu'il lui était arrivé de se servir dans ses articles de notes de son frère. Dans les années 1920, il continue à suivre le travail des formalistes de Petrograd, remarque positivement leur volume d'articles à la mémoire de Blok (parle même d'un "nouveau secteur de la science ») et participe à l'un des derniers recueils du mouvement, Fel'eton [Le feuilleton satirique], 1927, avec l'article « Fel'eton i esse » [Feuilleton satirique et essai] ${ }^{31}$. L'intitulé de ses cours montre aussi une influence formaliste : à côté de « Phonétique et stylistique du français ", on trouve "Théorie des genres littéraires", "Évolution du roman en 
Espagne ». Une de ses lettres à Viktor fait état d'un travail qui lui aurait été commandé par Tynjanov et Kazanskij sur l'histoire de la traduction. En lui cohabitaient une très bonne connaissance de la culture ancienne, médiévale et un intérêt pour les formes les plus modernes (argot).

13 L'autre figure liée au formalisme à ses débuts est Konstantin V. Močul'skij (1892-1948) ${ }^{32}$. Originaire d'Odessa, il fait ses études dans le même département que Vladimir Šklovskij, mais un peu plus tard, entre 1910 et 1914. Lui aussi connaissait un nombre impressionnant de langues romanes (français, italien, provençal, espagnol, portugais, catalan, roumain), les langues anciennes, grec et latin, et l'arabe. Sa spécialité est plus particulièrement la littérature italienne et française (Molière); il est attaché à l'université de SaintPétersbourg en 1914, mais, après la révolution, il retourne à Odessa d'où il émigre en 1920, lorsque la ville est prise par les Rouges. On le trouve d'abord en Bulgarie (il enseigne à l'université de Sofia entre 1920 et 1922), puis il se fixe à Paris. Dans l'émigration, il laissera des ouvrages de critique littéraire de qualité, consacrés à des classiques russes, Gogol', Dostoevskij, Belyj, étudiés sous l'angle de leur évolution spirituelle. Aussi a-t-on du mal à imaginer que dans sa jeunesse il ait pu être un adepte de l'étude formelle de la littérature. Pourtant, le titre d'une de ses études de jeunesse « La technique du comique chez Carlo Gozzi » (1916) est suffisamment éloquent. Močul'skij s'intéressait à toutes les questions formelles et aux problèmes de métrique. C'était un grand ami de Žirmunskij qui veut le faire recruter à Saratov en $1918^{33}$; dans l'exposé qu'il prépare en vue de son élection, il le caractérise, entre autres, comme " un représentant conscient et conséquent de la méthode formelle» (soznatel'nyj $i$ posledovatel'nyj predstavitel' formal'nogo metoda) et il ajoute que, dans ce domaine, il se trouve sous son influence. De son côté, Močul'skij a rédigé un compte rendu très élogieux des premiers recueils de l'Opojaz ${ }^{34}$, qui n'a pas été publié à l'époque et il déplorera ensuite d'être à Paris au moment où ces tendances connaissent leur plein essor en Russie.

Certes, les éléments de biographie formaliste de Vladimir Šklovskij et de Konstantin Močul'skij sont minces, mais ils nous autorisent néanmoins à les inclure dans le cercle large des formalistes (Vladimir Šklovskij figure, par exemple, dans la liste des membres de l'Opojaz ${ }^{35}$ ). Leur présence nous invite à rechercher dans les travaux de romanistique de l'époque des éléments qui auraient pu favoriser l'émergence d'une approche formelle de la littérature. Plus généralement, il s'agit, à travers leur exemple, de rappeler l'importance du département d'études romanes et germaniques de l'université de SaintPétersbourg dans la genèse du formalisme russe ${ }^{36}$.

15 Ce département est aussi important d'un autre point de vue. Il s'est trouvé accueillir, au début $\mathrm{du} \mathrm{xx}^{\mathrm{e}}$ siècle, un certain nombre de poètes, dont Nikolaj Gumilev et Osip Mandel'stam, ce qui contribua à créer une atmosphère bien particulière, propice à la réflexion méthodologique et créatrice. De fait, dans la variante pétersbourgeoise du formalisme russe, la conjonction de la philologie et de la poésie est capitale. Il ne s'agit pas de rappeler les liens bien connus entre formalisme et futurisme, ni même de souligner l'importance de la matrice acméiste, mais d'insister sur un trait de personnalité plus profond des philologues de l'Opojaz: tous ou presque écrivent des vers et sont fortement marqués par la poésie contemporaine ${ }^{37}$. Leur démarche scientifique elle-même est déterminée par leur nature d'artiste. 


\section{Science et intuition : "Nous nous sommes passés du Geist des Allemands ${ }^{38}$}

16 Aussi surprenant que cela puisse paraître, une partie importante de l'héritage des formalistes russes peut difficilement être qualifiée de "scientifique ${ }^{39}$. Leurs articles, publiés à la hâte dans des périodiques non académiques, pèchent par manque de rigueur ; les références ne sont pas toujours indiquées; le jargon employé est obscur, la terminologie incertaine. Certains philologues de Moscou que d'aucuns considèrent aujourd'hui comme les authentiques « formalistes » ne voulaient même pas les citer. Sur le tard, Viktor Žirmunskij a avoué à Viktor Šklovskij, qu'il n'avait jamais compris l'intérêt du livre de Jurij Tynjanov sur la sémantique du vers et qu'il considérait sa théorie des archaïstes comme erronée. Pourtant, on continue à lire et à commenter ces textes de Tynjanov comme d'autres publications de l'Opojaz. Il faut alors admettre que l'on se trouve face à une démarche originale, qui, nonobstant son caractère peu rigoureux, arrive néanmoins à des résultats.

Les membres de l'Opojaz, écrit Mixail Gasparov, partaient de la perception vivante du processus littéraire contemporain : par analogie avec lui, ils se représentaient la dynamique du processus littéraire des $\mathrm{XVIII}^{\mathrm{e}}$-XIX ${ }^{\mathrm{e}}$ siècles et ensuite reconstituaient la façon dont un texte aujourd'hui fossilisé avait pu être engendré par ce processus vivant. ${ }^{40}$

On comprend alors la part dominante jouée par l'intuition, qu'il s'agit de rendre vraisemblable par la persuasion plus que par la démonstration. Le principal est une nouvelle vision des textes, une nouvelle conception, étayée par deux-trois exemples. Cette démarche est assez contraire à la tradition philologique pour laquelle est scientifique ce qu'on peut décrire, puis vérifier de manière objective, à partir d'hypothèses de travail établies de manière rigoureuse. Ainsi définie, la science ne découvre pas, elle établit des faits. Dans ses travaux sur le théâtre français de l'époque classique, Boris Jarho confirme la validité de telle affirmation, circulant avant lui de façon empirique, mais il ne propose pas de nouvelle interprétation du classicisme ${ }^{41}$. «Pourquoi Tynjanov au moment de choisir sa voie s'est-il tourné vers l'Opojaz? La réponse est simple: il avait une bonne intuition littéraire et pas assez de cette assiduité pédante qui, après de longs et fastidieux calculs, confirme dans 9 cas sur 10 ce qui était visible à l'œil nu. $»^{42}$

18 Cet élément est essentiel à rappeler lorsqu'on aborde la question des sources étrangères du formalisme russe. Il est certes capital de l'intégrer à un ensemble européen plus large. Néanmoins, il est fondamental de commencer par distinguer les deux branches principales du mouvement, celle de Moscou (qualifiée le plus souvent de formalophilosophique) et celle de Pétersbourg-Petrograd. En ce qui concerne cette dernière, il ne faut pas perdre de vue d'autres clés de lecture, parfois plus productives, et, en premier lieu, tout ce qui renvoie au sens de l'écriture, au don artistique chez ses trois principaux représentants, šklovskij, Tynjanov, Ejhenbaum. Comme l'a montré Maksim Šapir, la formule de Tynjanov, « tesnota stihovogo rjada » (mot à mot : cohésion, resserrement de la série vers) pourrait bien venir d'un rapprochement (paronomase) entre les mots allemands dicht, serré et dichten, faire des vers, ce qui aurait donné la formule allemande * die Dichte der Gedichtreihe (mot à mot l'épaisseur, Dichte, de la série du poème, Gedicht), que Tynjanov aurait ensuite traduite en russe ${ }^{43}$. 
19 Cette façon de travailler explique aussi une des positions de principe du formalisme pétersbourgeois, son refus de toute théorisation préalable : ne pas commencer par des définitions, parachutées d'en haut, par la mise au point d'un modus operandi; les concepts esthétiques sont à déduire par en bas, de l'étude des faits et des faits strictement littéraires, c'est-à-dire de l'étude de textes précis, historiquement, sociologiquement déterminés. C'est là que réside l'explication de la fameuse déclaration de Tynjanov :

Nous nous sommes passés du Geist des Allemands et avons compris de quoi il

retournait. ${ }^{44}$

«Le Geist des Allemands » ne désigne pas des travaux particuliers, mais une façon de faire de la science ; pour Tynjanov et Ejhenbaum ${ }^{45}$, la science allemande commence par poser des prémisses philosophico-esthétiques et passe ensuite à leur application dans le domaine littéraire. Leur démarche est tout autre :

Le formalisme russe est intéressant en ce qu'il affirme l'autonomie des catégories esthétiques par en bas, à partir de l'étude du processus même de l'évolution historico-littéraire et de sa propre conception de ce qui est scientifique, et non par en haut (à partir de la sphère de la source éternelle du beau, de la sanction supérieure de l'art verbal ou d'une recherche spirituelle). ${ }^{46}$

On peut débattre indéfiniment du bien-fondé ou non de cette position de principe ; pour certains, elle est la source de la faillite du formalisme pétersbourgeois et de son inconsistance théorique ${ }^{47}$. Pour d'autres, elle reste encore aujourd'hui au contraire une affirmation salutaire. Elle explique en tout cas la cécité des formalistes de Moscou, refusant de citer les travaux de l'Opojaz ou les réticences de Viktor Žirmunskij. Mais la dimension artistique incontestable d'une partie au moins de l'héritage des formalistes nous invite aussi à relativiser l'importance de la question des sources étrangères ou tout au moins à la considérer d'une manière un peu différente. Il ne faut pas s'attendre à une trop grande rigueur dans l'utilisation de ces sources ; on ne doit peut-être pas y chercher la clé de leur travail, comme ils semblent nous le suggérer à travers la distinction établie entre genèse et tradition (évolution).

\section{La question des sources : genèse contre système}

Si nous avons insisté sur l'aspect artistique de l'héritage du formalisme pétersbourgeois, cela ne signifie pas cependant qu'il ait été incapable de rigueur scientifique. Une des règles, posée très tôt par les formalistes et en particulier par Tynjanov, et une règle qui garde aujourd'hui toute sa validité, est la distinction, nécessaire à pratiquer, entre ce qui relève des hasards, de la genèse et ce qui fait système, peut être analysé, décrit de façon objective et obéit à des lois (zakonomernosti) ${ }^{48}$.

La valeur heuristique de cette distinction a été montrée par Tynjanov sur l'exemple de traductions de Heine, réalisées par le poète russe Fedor Tjutčev. Si la genèse du texte de Tjutčev est bien un poème de Heine, le résultat est un poème de Tjutčev (système) qui ne peut s'expliquer que dans le "système " qui est le sien, à savoir la poésie de Tjutčev et plus largement l'évolution de la poésie russe du XIX ${ }^{e}$ siècle. Si l'on s'en tient à l'approche par la genèse, on manque le sens " évolutionnel » du texte et on l'interprète de manière réductrice ${ }^{49}$. C'est cette même distinction qui a poussé plus tard Tynjanov à se montrer sceptique vis-à-vis de l'« étude du quotidien de la littérature » (literaturnyj byt) de Boris Ejhenbaum, car là encore le risque était grand de considérer comme faisant partie du système des traits qui relevaient de la genèse accidentelle des phénomènes ${ }^{50}$. Si Tynjanov 
ne rejetait pas l'influence du hors-texte, il maintenait avec force la distinction entre " genèse » et « système » et une nécessaire hiérarchie des étapes qui ne devait envisager qu'en dernière instance le lien avec le hors-texte. Si ce projet est utopique et difficilement réalisable en pratique, dans les faits, il constitue une sérieuse mise en garde qui doit nous inciter à la plus grande prudence dans le travail sur les sources, qui, qu'on le veuille ou non, relèvent de la genèse. L'identification de sources, d'un contexte si tant est qu'elle soit exacte, ne nous livre pas encore forcément la clé d'un phénomène. En tout cas, la mise en évidence des sources ne doit pas faire perdre de vue la recherche de la qualité différentielle du mouvement, c'est-à-dire la façon dont, par l'assimilation, il a fait œuvre originale et ne proposait pas simplement une étude de la forme :

L'étude de la forme est une chose, la méthode formelle [russe] en tant que principe (en italique dans l'original) en est une autre, écrivait Ejhenbaum à Žirmunskij le 19 octobre 1921. Certes, nous étudions la forme depuis longtemps, indépendamment de l'Opojaz. Certes, il faut rappeler ici Veselovskij et même Petrov $^{51}$. Tout le département d'études romanes et germaniques nous a appris à aborder les questions de forme, sans compter la masse considérable de travaux savants dont nous nous occupons depuis longtemps. Mais Vitja [Žirmunskij], c'est tout à fait autre chose ! Dans tout ce dont je viens de parler, il y avait toujours l'idée que la forme était l'extérieur, qui recouvrait quelque chose d'autre et que c'est de ce quelque chose d'autre qu'il fallait parler en définitive..$^{52}$

Aussi peut-il sembler paradoxal de voir qu'aujourd'hui l'intérêt pour les formalistes russes se concentre presque exclusivement sur la question des "sources", des modèles, des emprunts, même s'il s'agit d'une étape de la recherche parfaitement justifiée. Il reste alors à souhaiter que leurs réflexions en ce domaine nourrissent davantage les travaux engagés en la matière.

\section{NOTES}

1. Victor Erlich, Russian Formalism : History, doctrine, The Hage - P. : Mouton, $3^{e}$ éd. 1969, chapitre « The Forerunners », p. 19-32.

2. Cf. par exemple Claude Lévi-Strauss, «La structure et la forme : réflexions sur un ouvrage de Vladimir Propp ", Anthropologie structurale, 2, P. : Plon, 1973, p. 139-173. Sur cette question, cf. la contribution récente de Frédérique Matonti, "L'anneau de Moebius : la réception en France des formalistes russes ", Actes de la recherche en sciences sociales, mars 2009, 176-177, p. 52-67.

3. Cf. Irina Popova, Kniga Bahtina o Fransua Rable i ee značenie dlja teorii literatury [Le livre de Bahtin sur François Rabelais et sa signification pour la théorie de la littérature], M. : Imli Ran, 2009.

4. Cf., principalement, Aleksandr Dmitriev, « Le contexte européen (français et allemand) du formalisme russe ", Cahiers du monde russe, 43 (2/3), 2002, p. 423-440 ; Sergej Zenkin, éd., Russkaja teorija [La Théorie russe], M. : RGGU, 2004 ; Céline Trautmann, éd., L’Allemagne des linguistes russes, P.: CNRS Éditions, Revue germanique internationale, (3) 2006 ; Ilona Svetlikova, Istoki russkogo formalizma : tradicija psihologizma i formal'naja škola [Les sources 
du formalisme russe : la tradition du psychologisme et l'école formelle], M. : NLO, 2005; Patrick Sériot, Structure et totalité: Les origines intellectuelles du structuralisme en Europe centrale et orientale, P.: PUF, 1999 ; Sergueï Tchougounnikov, Du «proto-phénomène » au phonème: le substrat morphologique allemand du formalisme russe, Kaliningrad : Université d'État de Kaliningrad, 2002 et le bloc thématique, "Filosofskie traektorii russkogo formalizma " [Les trajectoires philosophiques du formalisme russe], Novoe literaturnoe obozrenie (désormais NLO), 80, 2006, 4, p. 7-90, dont l'article de Zenkin sur les formalistes et Durkheim. Voir enfin les ouvrages collectifs: Céline Trautmann-Waller, Carole Maigné, éds., Formalismes esthétiques et héritage herbatien: Vienne, Prague Moscou, Hildesheim - Zürich - New York : Georg Olms Verlag, 2009 et Michel Espagne, Ekaterina Dmitrieva, Valerij Zemskov, éds., Evropejskij kontekst russkogo formalizma ( $k$ probleme éstetičeskih peresečenij: Francija, Germanija, Italija, Rossija) [Le Contexte européen du formalisme russe (croisements esthétiques : France, Allemagne, Italie, Russie)], M. : Imli Ran, 2009.

5. Le livre est désormais accessible en français, Pavel Medvedev, La Méthode formelle en littérature: introduction à une poétique sociologique (1928), tr. fr. par Bénédicte Vauthier et Roger Comtet, Toulouse : PUM, 2008, p. 139. Comme on sait, cet ouvrage est considéré comme reflétant pour une bonne part les idées de M.M. Bahtin.

6. Heinrich Wölfflin, Principes fondamentaux de l'histoire de l'art (1915); la lecture de cet ouvrage est attestée par Boris Ejhenbaum dans son Journal de 1919, "Je continue Wölfflin. Constamment apparaissent des analogies avec la construction de l'histoire de la littérature. Il faudrait établir un système de concepts fondamentaux et soumettre la littérature à l'analyse, en regardant un phénomène donné à chaque fois d'un seul point de vue, en extrayant tout ce qui s'y rapporte. », cité dans B. Ejhenbaum, O literature [De la littérature], M., 1987, p.511. Cf. également Ejhenbaum, "Théorie de la méthode formelle» [1925], Literatura, teorija, kritika, polemika [Littérature, théorie, critique, polémique], L.: Priboj, 1927, p. 118.

7. Ses travaux sur le roman anglais constituent une référence importante pour Ejhenbaum (lettre à Žirmunskij, «Perepiska Ejhenbaum i Žirmunskogo » (Correspondance entre Ejhenbaum et Žirmunskij), Tynjanovskij sbornik 3, Riga, p. 310 (désormais «Perepiska») et Molodoj Tolstoj [Le jeune Tolstoj], 1922, reprint W. Fink Verlag, 1968, p.148, note 52) ainsi que pour M.A.Petrovskij et A.A. Reformatskij à Moscou.

8. Cf. «Perepiska », lettre du 24 novembre 1917, p. 296, lettre du 5 août 1918, p. 306. Cf. également la bibliographie constituée par B. Ejhenbaum et publiée à la suite de son article «Comment est fait Le Manteau de Gogol ", Poètika : sborniki po teorii poétičeskogo jazyka, Petrograd, 1919, p. 167-168.

9. Raffaela Vassena, «K rekonstrukcii istorii dejatel'nosti Instituta živogo slova (1918-1924)» [Reconstitution de l'histoire de l'activité du l'Institut du mot vivant 1918-1924], et Ekaterina Choun, Craig Brandist, « Iz predistorii Instituta živogo slova: protokoly zasedanij kursov xudožestvennogo slova » [Préhistoire de l'Institut du mot vivant: procès-verbaux des réunions des cours d'art dramatique], NLO, 86 (4), 2007, p. 79-95 et p. 96-106.

10. Oskar Walzel, Impressionnisme et expressionisme dans l'Allemagne contemporaine 1890-1920, Petrograd, 1922 et Problème de la forme en poésie, 1923.

11. Voir aussi son article sur les courants contemporains de la science allemande, « Novejšie tečenija istoriko-literaturnoj mysli v Germanii » [Les courants contemporains 
de la pensée historico-littéraire en Allemagne], Poètika, 2, 1927, repris dans Iz istorii zapadnyh literatur [Histoire des littératures occidentales], L., 1981.

12. Zeitschrift für slavische Philologie, Bd 1, H 1-2, 1925, p. 117-152. L'article est désormais accessible en français, "Les problèmes formels dans la science russe de la littérature ", tr. fr. Roger Comtet, Slavica occitania, 25, 2007, p. 225-284. Cf. du même auteur, « Viktor Maksimovitch Jirmounski (1891-1971) et le formalisme russe», p. 205-223 et "Viktor Maksimovič Žirmunskij (1891-1971), passeur de cultures entre Russie et Allemagne », L'Allemagne des linguistes russes, p. 111-125.

13. Auteur de Philosophie der Kunst, 1909, traduit en russe en 1911. Cf. "Perepiska", journal 20 août 1918, p. 264, lettre du 9 juillet 1916, p. 284. Sur ce sujet, cf. Leonid Heller, " Des signes et des fleurs, ou Victor Chklovski, Broder Christiansen et la "sémiologie formaliste" ", De la littérature russe: Mélanges en l'honneur de Michel Aucouturier, P. : IES, 2005, p. 201-215.

14. Cf. Medvedev, La Méthode formelle en littérature, p. 158: "La véritable patrie de la méthode formelle dans la science de la littérature est bien évidemment la France ».

15. Cf. M.O. Čudakova, E.A. Toddes, « La première traduction russe du Cours de linguistique générale et l'activité du Cercle linguistique de Moscou ", Cahiers Ferdinand Saussure, 36, 1982 et Catherine Depretto, "Diffusion et réception du Cours de linguistique générale dans l'URSS des années vingt ", Actes du IV colloque de linguistique russe, P. - Toulouse, 1986, p. 77-93.

16. J.M. Curtis, "Bergson and Russian Formalism », Comparative Literature, XXVIII (2), Spring 1976, p. 109-121.

17. Cette question est devenue particulièrement dangereuse dans l'après-guerre lorsque avec le déchaînement du nationalisme et de l'antisémitisme d'État, entre 1946 et 1953, il était interdit de trouver la moindre origine étrangère à tout phénomène de la culture et de la science russes. On connaît la boutade qui circulait alors : « La Russie est le pays des éléphants ».

18. Catherine Depretto, «La question du formalisme moscovite ", Revue des études slaves, LXXVII, (1-2), 2008, p. 87-101.

19. Voir en priorité D.V. Ustinov, « Formalizm i mladoformalisty » [Formalistes et néoformalistes], stat'ja 1, NLO, 50 (4), 2001, p. 296-321 et St. Savickij « Spor s učitelem : načalo literaturnogo/issledovatel'skogo proekta L. Ginzburg " [Querelle avec son maître: le début du projet littéraire et de recherche de L. Ginzburg], NLO, 82 (6), 2006, p. 129-154.

20. Cf. entre autres K. Postoutenko "Pis'ma B.V. Tomaševskogo k S.P. Bobrovu " [Lettres de Tomaševskij à S.P.Bobrov], Pjatye Tynjanovskie čtenija: tezisy dokladov $i$ materialy dlja obsuždenija, Riga: Zinatne, 1990, p.133-148; du même auteur, "Iz materialov po istorii stihovedenija v Rossii » [Matériaux pour l'histoire de l'étude du vers en Russie], Tynjanovskij sbornik (Pjatye Tynjanovskie čtenija) Riga - M.: ZinatneImprint, 1994, p. 305-322 ainsi que "Biografija i filologičeskaja dejatelnost' N.V. Nedobrovo " [Biographie et activité philologique de N.V.Nedobrovo], articles de R. Timenčik, I.G. Kravcova, G.V. Obatnin, N.I. Krajceva, S.V. Šumihin, K.Ju. Postoutenko, M.L. Gasparov, Šestye Tynjanovskie čtenija : tezisy dokladov i materialy dlja obsuždenija), RigaM., 1992, p. 82-152. Voir enfin K. Postoutenko éd., "G.A. Šengeli : svobodnyj stih» [G.A. Šengeli : le vers libre], ibid., p. 269-300 et M.L. Gasparov, « G.A. Šengeli : ot iskusstva k nauke » (G.A. Šengeli : de l'art à la science), Pjatye Tynjanovskie čtenija, 1994, p. 323-334. 
21. Ce qui ne signifie pas qu'il n'y ait pas eu ailleurs des cours sur la littérature européenne et sur la littérature italienne en particulier, cf. par exemple à Moscou les cours sur Dante de N.I.Storoženko (édités en 1891) ou de E.F. Braun (1866-1918, "moskovskij Braun») entre 1899 et 1914; Braun serait en particulier l'auteur d'une traduction non publiée de Vita Nuova de Dante.

22. Pour se faire une idée de l'activité de la société, on pourra consulter la série de ses bulletins, Zapiski neofilologičeskogo obščestva, I, 1888 ; II, 1892 ; III, 1894 ; IV, 1910 ; V, 1911 ; VI, 1912 ; VII, 1914 ; VIII, 1915. Cf. en particulier, IV, 1910, p. 1-12.

23. On pourrait en ce domaine reprendre ce qu'a fait Oleg Lekmanov pour l'acméisme et introduire la notion de " cercle » (cf. sa notion de « cercle de Gumilev »); c'est-à-dire montrer l'existence de différents cercles, plus ou moins étendus, autour de Šklovskij ou d'autres personnalités et dont la composition a pu varier.

24. Son arrestation de 1929, est liée au démantèlement d'un cercle privé d'études romanes, tenu par la sœur de l'hispanisant K.D. Petrov, "Le foyer familial français » («Francuzskij semejnyj očag»), auquel participaient entre autres l'historien de la France, N.I. Kareev, B.A. Krževskij, le poète V. Pjast... Ce démantèlement est à rattacher à la vague de répressions qui frappe l'intelligentsia et en particulier tous les cercles privés au moment du grand tournant de 1928-1932, cf. par exemple l'arrestation de M.M. Bahtin, liée au démantèlement du cercle de A.A. Mejer, « Résurrection ».

25. La participation de son frère au recueil d'écrivains sur ce chantier du Goulag (1933) prend ainsi une autre dimension; Viktor Šklovskij ne serait pas allé sur le chantier avec la brigade d'écrivains, mais seul, selon A. Galuškin, précisément pour voir son frère (récit différent chez L. Stepanova). Quoi qu'il en soit, tous les documents conservés dans ses archives montrent que Viktor s'est toujours occupé de son frère et a tout fait pour le sortir de camp. Ignorant tout de son sort, il a, en particulier, entrepris de nouvelles démarches en 1939-1940 (le dossier contient des attestations de V.Žirmunskij, V. Šismarev, O. Forš, Ju. Tynjanov...).

26. Entre 1922 et 1925, il était au camp des îles Solovki. La date exacte de son exécution n'a été connue que lorsque ont été publiées les listes de fusillés (rasstrel'nye spiski), en 1996 d'abord, puis dans Leningradskij martirolog 1937-1938 (Martyrologe de Leningrad 1937-1938), t. 3, nov. 1937, SPb. : Rossijskaja nacional'naja biblioteka, 1998, p. 456.

27. On peut consulter cet ouvrage à la bibliothèque des Langues orientales à Paris. Compte rendu assez critique par L.N. Lunc (traduction trop consciencieuse et pas d'appareil critique), publié dans Kniga i revoljucija, 6 (18), 1922, p. 51-52. Suivant le spécialiste italien Pio Rajna, Šklovskij préfère « eloquio » à « eloquentia », plus tardif, mais communément retenu aujourd'hui, en particulier en France.

28. RGALI (Rossijskij gosudarstvennyj arhiv literatury i iskusstva - Archives russes d'État de littérature et d'art), f. 562, opis' 2, ed. hr. 922, 266 feuillets. Ce qui reste des papiers de Vladimir se trouve dans le fonds de son frère au RGALI (Moscou). Sur Vladimir Šklovskij, cf. l'article de L.G. Stepanova, in Leningradskij martirolog 1937-1938, p. 547-549 ainsi que L.G. Stepanova, D.V. Ustinov « O sud'be Vladimira Borisoviča Šklovskogo [Du destin de V.B. Šklovskij]», in N.N. Kazanskij éd., Materialy konferencii, posvjaščennoj 110letiju so dnja roždenija akademika Viktora Maksimoviča Žirmunskogo [Conférence dédiée au $110^{\mathrm{e}}$ anniversaire de la naissance de l'académicien V.M. Žirmunskij : documents], SPb. : Nauka, 2001, p. 29-36 et surtout L.G.Stepanova, "Vladimir Borisovič šklovskij (1889-1937) », Tynjanovskij sbornik, vypusk 13, M. : Vodolej, 2009, p. 535-557. 
29. Dmitrij Konstantinovic Petrov (1872-1925): disciple et successeur de Veselovskij, professeur au département d'études romanes et germaniques de l'université de SaintPétersbourg avant la révolution, romaniste et surtout hispaniste. Il a laissé le souvenir d'un érudit et d'un bon pédagogue, même s'il ne s'illustrait pas par une grande originalité méthodologique.

30. Sborniki po teorii poétičeskogo jazyka, 1, 1916 et 2,1917.

31. Une note sur le même sujet est parue dans Novyj Lef, "Literaturnyj opyt v ego formal'nom okruženii » [L'expérience littéraire et son entourage formel], 1927, 7, p. 39-49.

32. Cf. la publication capitale et richement commentée des lettres de K.V. Močul'skij à V.M. Žirmunskij par A.V Lavrov, NLO, 35, 1999, p. 117-131. Renseignements sur le cercle d'études romanes et germaniques, p. 146, 149, 153-154, 172.

33. Ce n'est finalement pas lui qui sera choisi mais un romaniste de Moscou, N.S. Arsen'ev. L'ouvrage de Žirmunskij, Valerij Brjusov i nasledie Puškina [Valerij Brjusov et l'héritage de Puškin, 1922] a été écrit avec l'aide de Močul'skij. Cf. sa lettre à Ejhenbaum à ce sujet, « Perepiska », p. 303-304.

34. NLO, 35, 1999, p. 212-214.

35. Cf. A.V. Krusanov, "Opojaz », in Russkij avangard 1907-1932 : istoričeskij obzor, t. 2, Futurističeskaja revoljucija 1917-1921 [L'avant-garde russe, 1907-1932 : panorama historique, t. 2, La révolution futuriste, 1917-1921], kn. 1, M. : NLO, 2003, p. 297, 301.

36. Ce qui est parfaitement attesté dans les documents suivants : lettre de Ejhenbaum à Žirmunskij du 19-10-1921, «Perepiska», p.313-314, ou introduction de Viktor Žirmunskij à Voprosy teorii literatury: stat'i 1916-1926 [Questions de théorie de la littérature : articles 1916-1926], L. : Academia, 1928, p. 8.

37. Medvedev, La Méthode formelle en littérature, «Mais le milieu qui a vraiment nourri le formalisme dans la première phase de son développement fut la poésie contemporaine " (p.165) ou encore « le culte des mots des acméistes, déjà quelque peu atténué est plus proche en tant que tel des formalistes que celui des symbolistes », (p. 168).

38. Lettre de Tynjanov à Šklovskij, de février-mars 1928, PILK, p. 536.

39. Cf. Sergej Zenkin, "Otkrytie byta russkimi formalistami» [La découverte du quotidien par les formalistes russes], Lotmanovskij sbornik, 3, M. : OGI, 2004, p. 806-821.

40. M.L. Gasparov, « Naučnost' i xudožestvennost' v tvorčestve Tynjanova " [Caractère scientifique et caractère artistique dans l'œuvre de Tynjanov], Četvertye Tynjanovskie čtenija, Riga : Zinatne, 1990, p. 14.

41. Gasparov : « Jarho s'appuyait sur les méthodes, établies par le positivisme, à partir de l'étude du folklore, des textes de l'antiquité et du Moyen Âge : la mise en évidence de traits caractéristiques, l'étude statistique ; la systématisation avec pour résultat d'abord une description statistique de textes séparés et ensuite la reconstitution d'un processus général. » Ibid., p. 14.

42. Ibid., p. 14.

43. Šapir, «Anketa k 100-letiju so dnja roždenija Tynjanova » [Questionnaire pour les cent ans de la naissance de Tynjanov], Tynjanovskij sbornik, 9, Riga-M., 1995-1996, p. 68-69.

44. Lettre de Tynjanov à Šklovskij, de février-mars 1928, PILK, p. 536. "Le "Geist" (l'esprit) des Allemands " est peut-être une allusion à la Phénoménologie de l'esprit de Hegel et à la tradition esthétique et philosophique allemande. Elle vise aussi celui qui 
dans l'environnement scientifique immédiat incarne cette "science allemande", V. Žirmunskij. À ce sujet, cf. A. Dmitriev, « Le contexte européen (français et allemand) $\mathrm{du}$ formalisme russe", ainsi que du même auteur «Polemika V.M. Žirmunskogo s formal'noj školoj i nemeckaja filologija » [La philologie allemande dans la polémique entre Žirmunskij et l'école formelle], in Kazanskij éd., Materialy konferencii, p. 66-74.

45. Cf. ses déclarations contre le formalisme de Moscou (recueils Ars poetica) qui ne reconnaît que la « science allemande ». Ce motif réapparaît au moment du Dégel dans sa méfiance à l'égard des études qui semblent renouer avec les études formelles et utilisent sans distance critique des termes engendrés par la philosophie allemande (romantisme, réalisme, forme, contenu), lettre à Aleksandr Slonimskij, publiée par E.A.Toddes, «B.M. Ejhenbaum v 30-50-e gody " [B.M.E. dans les années 1930-1950], Tynjanovskij sbornik, vyp. 11, M.: OGI, 2002, p. 681.

46. A. Dmitriev, «Ėstetičeskaja avtonomija i istoričeskaja determinacija: russkaja gumanitarnaja teorija pervoj treti $\mathrm{xx} v \mathrm{v}$ v svete problematiki sekuljarizacii » [Autonomie esthétique et détermination historique: la théorie russe en sciences humaines du premier tiers du $\mathrm{xx}^{\mathrm{e}}$ à la lumière de la problématique de la sécularisation], in Zenkin, éd., Russkaja teorija, p. 35.

47. Cf. par exemple la lettre de Pasternak à Medvedev (20 août 1929), citée d'après Catherine Depretto éd., L'Héritage de Bakhtine, Bordeaux : PUB, 1996, p. 176-177.

48. Voir M.O. Čudakova, « K ponjatiju genezisa » [Le concept de genèse], Revue des études slaves, LV (3), 1983, p. 409-418, repris dans Tynjanovskij sbornik 2, Riga - M., 1986, p. 103-131 et dans Literatura sovetskogo prošlogo [La littérature du passé soviétique], M., 2001, p. 433-453.

49. Cf. "Tiouttchev et Heine", in Iouri Tynianov, Formalisme et histoire littéraire, présentation, traduction, commentaires de C. Depretto, Lausanne, L'Âge d'homme, 1987, p. 182-195.

50. Cf. comment Ejhenbaum «dérape » lorsqu'il découvre l'existence du travail de Proudhon, intitulé La Guerre et la paix et qu'il y voit la clé de Guerre et paix de Tolstoj; la nécessité de maintenir la distinction entre genèse et tradition est explicitée, en ce cas précis, par la phrase de Šklovskij : « un traité politique ne peut pas être la source d'un roman ".

51. Cf. note 29.

52. « Perepiska », p. 313.

\section{RÉSUMÉS}

Résumé

L'article retrace l'historique de la question des sources du formalisme russe et met en évidence les difficultés propres à cette question. Le formalisme russe étant divers, il est difficile de parler de ses sources en général. Ainsi, si l'on prend l'exemple de deux formalistes oubliés, Vladimir Šklovskij et Konstantin Močulskij, doit-on intégrer, à ces sources, leur domaine de spécialité, les 
études romanes. Plus généralement, il y a dans le courant pétersbourgeois du formalisme russe une forte dimension intuitive qui rend aléatoire toute recherche d'emprunts conceptuels rigoureux. On rappelle pour finir que les formalistes eux-mêmes (Tynjanov en particulier) ont tenté avec la distinction entre genèse et évolution de fournir aux chercheurs des outils susceptibles de les aider dans les problématiques des sources et des transferts.

Abstract

The article traces the history of the question of the sources of Russian Formalism and stresses the difficulties inherent to this question. Russian Formalism is a diverse movement, and it is difficult to speak of its sources as a whole. For instance, if we take the example of two forgotten Formalists, Vladimir Shklovskii and Konstantin Mochulskii, are we to consider their field of research, Romance languages, as one of the sources of Formalism? More generally, there is in the Petersburg strand of Formalism a strong intuitive dimension that makes the search for rigorous conceptual borrowings uncertain. Last, it must be known that the Formalists themselves (particularly Tynianov) tried to use the distinction between genesis and evolution to provide scholars with tools liable to help them deal with questions relative to sources and transfers.

\section{AUTEUR}

\section{CATHERINE DEPRETTO}

Centre de recherches sur les cultures et littératures d'Europe centrale, orientale et balkanique, EA 4084, Paris-Sorbonne 